\title{
Comparison of language cortex reorganization patterns between cerebral arteriovenous malformations and gliomas: a functional MRI study
}

\author{
Xiaofeng Deng, MD, ${ }^{1}$ Yan Zhang, MD, PhD, ${ }^{1}$ Long Xu, MD, PhD, ${ }^{1}$ Bo Wang, PhD, ${ }^{2}$ \\ Shuo Wang, MD, PhD, ${ }^{1}$ Jun Wu, MD, PhD, ${ }^{1}$ Dong Zhang, MD, PhD, ${ }^{1}$ Rong Wang, MD, PhD, ${ }^{1}$ \\ Jia Wang, MD, ${ }^{1}$ and Jizong Zhao, MD, PhD'1
}

1Department of Neurosurgery, Beijing Tiantan Hospital, Capital Medical University; and ${ }^{2}$ State Key Laboratory of Brain and Cognitive Science, Beijing MRI Center for Brain Research, Institute of Biophysics, Chinese Academy of Sciences, Beijing, China

\begin{abstract}
OBJECT Cerebral arteriovenous malformations (AVMs) are congenital malformations that may grow in the language cortex but usually do not lead to aphasia. In contrast, language dysfunction is a common presentation for patients with a glioma that involves language areas. The authors attempted to demonstrate the difference in patterns of language cortex reorganization between cerebral AVMs and gliomas by blood oxygen level-dependent (BOLD) functional MRI (fMRI) evaluation.
\end{abstract}

METHODS The authors retrospectively reviewed clinical and imaging data of 63 patients with an unruptured cerebral AVM (AVM group) and 38 patients with a glioma (glioma group) who underwent fMRI. All the patients were right handed, and all their lesions were located in the left cerebral hemisphere. Patients were further categorized into 1 of the 2 following subgroups according to their lesion location: the BA subgroup (overlying or adjacent to the inferior frontal or the middle frontal gyri [the Broca area]) and the WA subgroup (overlying or adjacent to the supramarginal, angular, or superior temporal gyri [the Wernicke area]). Lateralization indices of BOLD signal activations were calculated separately for the Broca and Wernicke areas. Statistical analysis was performed to identify the difference in patterns of language cortex reorganization between the 2 groups.

RESULTS In the AVM group, right-sided lateralization of BOLD signal activations was observed in 23 patients (36.5\%), including 6 with right-sided lateralization in the Broca area alone, 12 in the Wernicke area alone, and 5 in both areas. More specifically, in the 34 patients in the AVM-BA subgroup, right-sided lateralization of the Broca area was detected in 9 patients $(26.5 \%)$, and right-sided lateralization of the Wernicke area was detected in $4(11.8 \%)$; in the 29 patients in the AVM-WA subgroup, $2(6.9 \%)$ had right-sided lateralization of the Broca area, and $13(44.8 \%)$ had right-sided lateralization of the Wernicke area. In the glioma group, 6 patients (15.8\%) showed right-sided lateralization of the Wernicke area, including 2 patients in the glioma-BA subgroup and 4 patients in the glioma-WA subgroup. No patient showed right-sided lateralization of the Broca area. Moreover, although the incidence of right-sided lateralization was higher in cases of low-grade gliomas (5 in 26 [19.2\%]) than in high-grade gliomas (1 in 12 [8.3\%]), no significant difference was detected between them $(p=0.643)$. Compared with the AVM group, the incidence of aphasia was significantly higher $(p<0.001)$, and right-sided lateralization of language areas was significantly rarer $(p=0.026)$ in the glioma group.

CONCLUSIONS Right-sided lateralization of BOLD signal activations was observed in patients with a cerebral AVM and in those with a glioma, suggesting that language cortex reorganization may occur with both diseases. However, the potential of reorganization in patients with gliomas seems to be insufficient compared with patients AVMs, which is suggested by clinical manifestations and the fMRI findings. Moreover, this study seems to indicate that in patients with an AVM, a nidus near the Broca area mainly leads to right-sided lateralization of the Broca area, and a nidus near the Wernicke area mainly leads to right-sided lateralization of the Wernicke area.

http://thejns.org/doi/abs/10.3171/2014.12.JNS14629

KEY WORDS cerebral arteriovenous malformations; glioma; language cortex reorganization; functional MRI; aphasia; vascular disorders; diagnostic and operative techniques 


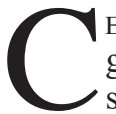
EREBRAL arteriovenous malformations (AVMs) are generally considered to be congenital vascular lesions that are characterized by direct communication of arteries to abnormal veins without interposing capillaries., ${ }^{2,5,15}$ Although AVMs may grow in eloquent areas such as the language cortex, patients with an AVM usually do not present with neurological deficits unless it has ruptured. It has been postulated that when these lesions develop in the usual anatomical sites of eloquent cortex, neuroplasticity will result in cortical reorganization of the functional areas, with displacement to other regions. Therefore, function will not be impaired. ${ }^{1}$

Unlike AVMs, most gliomas are thought to be acquired lesions, and the duration of illness is relatively short. Aphasia is a common presentation for patients with a glioma that involves language areas, and right-sided language dominance in right-handed patients is seldom reported. ${ }^{4,20,25}$

Although language cortex reorganization in patients with an AVM has been studied, previous reports have focused on global lateralization indices (LIs) as a measure of language dominance. However, it has been reported that the Broca and Wernicke areas can be lateralized asymmetrically in the setting of intracranial lesions. ${ }^{12,19,24}$ In such a case, studying the global LI alone, which could lead to wrong conclusions regarding language lateralization, might be inappropriate, because the activated voxels in different hemispheres will offset each other according to the formula of the LI. Therefore, we assessed the Broca and Wernicke areas separately in this study. Little is known about the difference in patterns of language cortex reorganization between patients with an AVM and those with a glioma.

In the clinic, blood oxygen level-dependent (BOLD) functional MRI (fMRI) is a useful and noninvasive method of mapping the location of eloquent areas. ${ }^{12}$ It offers topographic maps of language function with very high sensitivity and acceptable specificity, even in patients with vascular malformations. .,17 $^{3}$

In this study, we applied fMRI to study BOLD signal activations to evaluate language lateralization in 63 patients with an unruptured AVM and 38 patients with a glioma. In each type of case, LIs were calculated separately for the Broca and Wernicke areas, and the differences in language lateralization between the patients with an AVM and those with a glioma were studied systemically.

\section{Methods}

\section{Patient Population}

After the study was approved by the institutional review board of Beijing Tiantan Hospital, Capital Medical University, we retrospectively reviewed the medical records and imaging studies of 63 patients with a cerebral AVM (AVM group) and 38 patients with a glioma (glioma group) who underwent fMRI for preoperative evaluation between 2006 and 2013. All the patients were right handed, and their lesions were located in the frontal, temporal, or parietal lobe of the left hemisphere. Patients with an AVM and a history of rupture and hemorrhage were excluded from the study. All the patients were treated surgi- cally, and their lesions were confirmed by pathology examinations.

In the AVM group, there were 31 male and 32 female patients. Their ages ranged from 11 to 57 years (mean 31 years). According to the Spetzler-Martin grading system, there were 16 patients with Grade I, 23 with Grade II, 22 with Grade III, and 2 with Grade IV AVMs. The main clinical presentations included seizure in 43 patients, headache in 10, weakness of limbs in 2, aphasia in 3, and visual field deficits in 1; 4 patients were asymptomatic.

The glioma group enrolled 24 male and 14 female patients, and the mean age was 43 years (range $20-76$ years). According to the World Health Organization (WHO) brain tumor grading system (2007), ${ }^{8} 10$ patients were diagnosed pathologically with diffuse astrocytoma (WHO Grade II), 3 with oligodendroglioma (WHO Grade II), 12 with oligoastrocytoma (WHO Grade II), 1 with pleomorphic xanthoastrocytoma (WHO Grade II), 3 with anaplastic astrocytoma (WHO Grade III), 1 with anaplastic oligodendroglioma (WHO Grade III), 1 with anaplastic oligoastrocytoma (WHO Grade III), and 7 with glioblastoma (WHO Grade IV). The duration of the symptoms ranged from 1 to 32 months (mean [ \pm SD] $7 \pm 3$ months). Fourteen patients presented with aphasia, 9 with headache, 9 with seizure, 4 with weakness of limbs, and 2 with paresthesia.

The patients were further categorized into 1 of the 2 following subgroups according to the location of their lesion: the BA subgroup (patients with a lesion involving the frontal lobe, overlying or adjacent to the inferior frontal or middle frontal gyri [the Broca area]) and the WA subgroup (patients with a lesion involving the temporal or parietal lobe, overlying or adjacent to the supramarginal, angular, or superior temporal gyri [the Wernicke area]). If a lesion occupied more than 1 lobe, its location was defined according to the location occupied by the largest proportion of the lesion. According to these criteria, there were 34 patients $(54.0 \%)$ in the AVM-BA subgroup, 29 (46.0\%) in the AVM-WA subgroup, $24(63.2 \%)$ in the glioma-BA subgroup, and $14(36.8 \%)$ in the glioma-WA subgroup.

\section{fMRI Acquisition}

A Siemens Medical Systems Trio 3.0-T MRI system equipped at the Beijing Brain MRI Center was used for fMRI signal acquisition. Each subject underwent localizing imaging followed by T2-weighted structural imaging; a T2-weighted functional image was acquired at the same location (T2-weighted gradient echo, echo-planar imaging, TR $2000 \mathrm{msec}$, TE $29 \mathrm{msec}$, flip angle 90 , FOV 220 $\times 220 \mathrm{~mm}^{2}$, matrix $64 \times 64$, voxel size $3.4 \times 3.4 \times 4.0$ $\mathrm{mm}^{3}$ ); finally, a T1-weighted 3D fine-structure image was acquired (TR $3560 \mathrm{msec}$, TE $5 \mathrm{msec}$, flip angle 90, FOV $256 \times 256 \mathrm{~mm}^{2}$, matrix $256 \times 256$, slice thickness $1.0 \mathrm{~mm}$, voxel size $1.0 \times 1.0 \times 1.0 \mathrm{~mm}^{3}$ ).

Informed consent to local institutional review board regulations was obtained from each patient. The patients were given a silent reading task to perform, in which they were asked to silently read the Chinese characters presented on a screen (one by one), to consider the pronunciation for each character, and to understand the meaning of each character, word, or sentence. The stimulus was a paragraph composed of Chinese characters. On the ba- 
sis of previous findings of greater regularity effects for higher-frequency words than for lower-frequency words, highly familiar Chinese words with a frequency of occurrence of no fewer than 25 per million (according to the Modern Chinese Frequency Dictionary) were used in this study. ${ }^{21,22}$ The stimuli were shown through an LED projector system. During the paradigm, a set of 2 trials (30 seconds each) was performed and interleaved with 3 control conditions (20 seconds each), in which the patient was instructed to maintain fixation on a crosshair. Before scanning, the patients were trained with the paradigm using stimuli different than those presented during the protocol, and they demonstrated their ability to perform the task.

\section{fMRI Data Analysis}

fMRI data were processed on a Matlab 7.0 workstation (MathWorks) using SPM 8 (London University) and xjView toolbox. Maps were generated by identifying every voxel that exceeded a predetermined significance threshold $(\mathrm{p}<0.001)$ and overlaying these active voxels on T1-weighted structural images to display the anatomical location of brain activity.

Language cortex reorganization was studied in the Broca (inferior frontal and middle frontal gyri) and Wernicke (supramarginal, angular, and superior temporal gyri) areas separately. ${ }^{19}$ The LI was used to quantify the degree of lateralization of BOLD signal activations. It was calculated for the Broca and Wernicke areas separately using the following formula: $\mathrm{LI}=(\mathrm{VL}-\mathrm{VR}) /(\mathrm{VL}$ $+\mathrm{VR}$ ), where VL is the number of voxels activated in the left hemisphere and VR is the number of voxels activated in the right hemisphere. Language activation was categorized as 1 of 3 patterns. An LI of less than or equal to -0.2 was considered right-sided lateralization of BOLD signals, an LI of greater than or equal to 0.2 seconds was considered left-sided lateralization, and an LI between -0.2 and 0.2 suggested no clear hemispheric preference. ${ }^{12}$

For patients with an AVM, the lesion size, deep drainage veins, and Spetzler-Martin grade were determined from digital subtraction angiography and MRI. Lesion size was defined by the greatest diameter of the nidus.

fMRI data were used for intraoperative neuronavigation, and all 63 of the patients with an AVM and all 38 of the patients with a glioma were treated surgically.

\section{Statistical Analysis}

All statistical analyses were performed with SPSS (Windows version 18.0; IBM). The chi-square test for the
$\mathrm{R}$ by $\mathrm{C}$ (row by column) contingency tables was performed to analyze the difference in BOLD signal lateralization between the AVM group and the glioma group, and lateralization of the Broca and Wernicke areas was analyzed separately. Differences between the BA and WA subgroups were analyzed further. In addition, the incidence of language dysfunction was also compared between the 2 groups using the chi-square test. Odds ratios with $95 \%$ CIs are presented. A $p$ value of $<0.05$ was considered statistically significant.

\section{Results \\ AVM Group Analysis}

In the AVM group, right-sided BOLD signal lateralization was observed in 23 patients $(36.5 \%)$ (Table 1). Five patients had right-sided lateralization in both the Broca area $(\mathrm{LI}=-0.48 \pm 0.24)$ and Wernicke area $(\mathrm{LI}=-0.41 \pm$ 0.14; Fig. 1), 6 had right-sided lateralization in the Broca area alone ( $\mathrm{LI}=-0.40 \pm 0.22)$, and 12 had right-sided lateralization in the Wernicke area alone $(\mathrm{LI}=-0.74 \pm 0.24$; Fig. 2). Lesion size $(p=0.620)$, deep drainage veins $(p=$ $0.435)$, and Spetzler-Martin grade $(\mathrm{p}=0.532)$ were not significantly associated with right-sided lateralization.

In the BA subgroup, 10 (29.4\%) of 34 patients had rightsided BOLD signal lateralization; 6 patients had right-sided lateralization in the Broca area alone, 1 in the Wernicke area alone, and 3 in both areas (Fig. 1). Therefore, rightsided lateralization of the Broca area occurred in 9 patients (26.5\%), and right-sided lateralization of the Wernicke area occurred in 4 patients $(11.8 \%)$.

In the WA subgroup, 13 (44.8\%) of 29 patients had rightsided BOLD signal lateralization; 11 patients $(37.9 \%)$ had right-sided lateralization in the Wernicke area alone (Fig. 2 ), and 2 patients $(6.9 \%)$ had right-sided lateralization in both areas. No patient had right-sided lateralization in the Broca area alone. Thus, right-sided lateralization of the Broca area was observed in 2 patients (6.9\%), and rightsided lateralization of the Wernicke area was noted in 13 patients $(44.8 \%)$.

Moreover, we also studied the difference in right-sided lateralization of BOLD signal activations of language areas between the BA and WA subgroups. The results show that the incidence of right-sided activation of the Broca area was significantly higher in the BA subgroup (26.5\%) than in the WA subgroup (6.9\%) $\left(\chi^{2} 4.161, \mathrm{p}=0.041\right.$, OR 4.860 [95\% CI 0.956-24.703]). Similarly, the incidence of right-sided activation of the Wernicke area was significantly higher in the WA subgroup (44.8\%) than in the BA

TABLE 1. Number of patients with right lateralization of BOLD signal activations in the AVM and glioma groups

\begin{tabular}{cccccc}
\hline & & \multicolumn{4}{c}{ Rt-Sided Lateralization of BOLD Signal Activations* } \\
\cline { 3 - 6 } Group & Subgroup & Broca Area Alone & Wernicke Area Alone & Broca \& Wernicke Areas & None \\
\hline AVM $(n=63)$ & BA $(n=34)$ & 6 & 1 & 3 & 2 \\
\hline \multirow{2}{*}{ Glioma $(n=38)$} & WA $(n=29)$ & 0 & 11 & 0 & 16 \\
\hline \multirow{2}{*}{ Total $(n=101)$} & WA $(n=24)$ & 0 & 2 & 0 & 22 \\
\hline
\end{tabular}

* Values represent number of patients. 

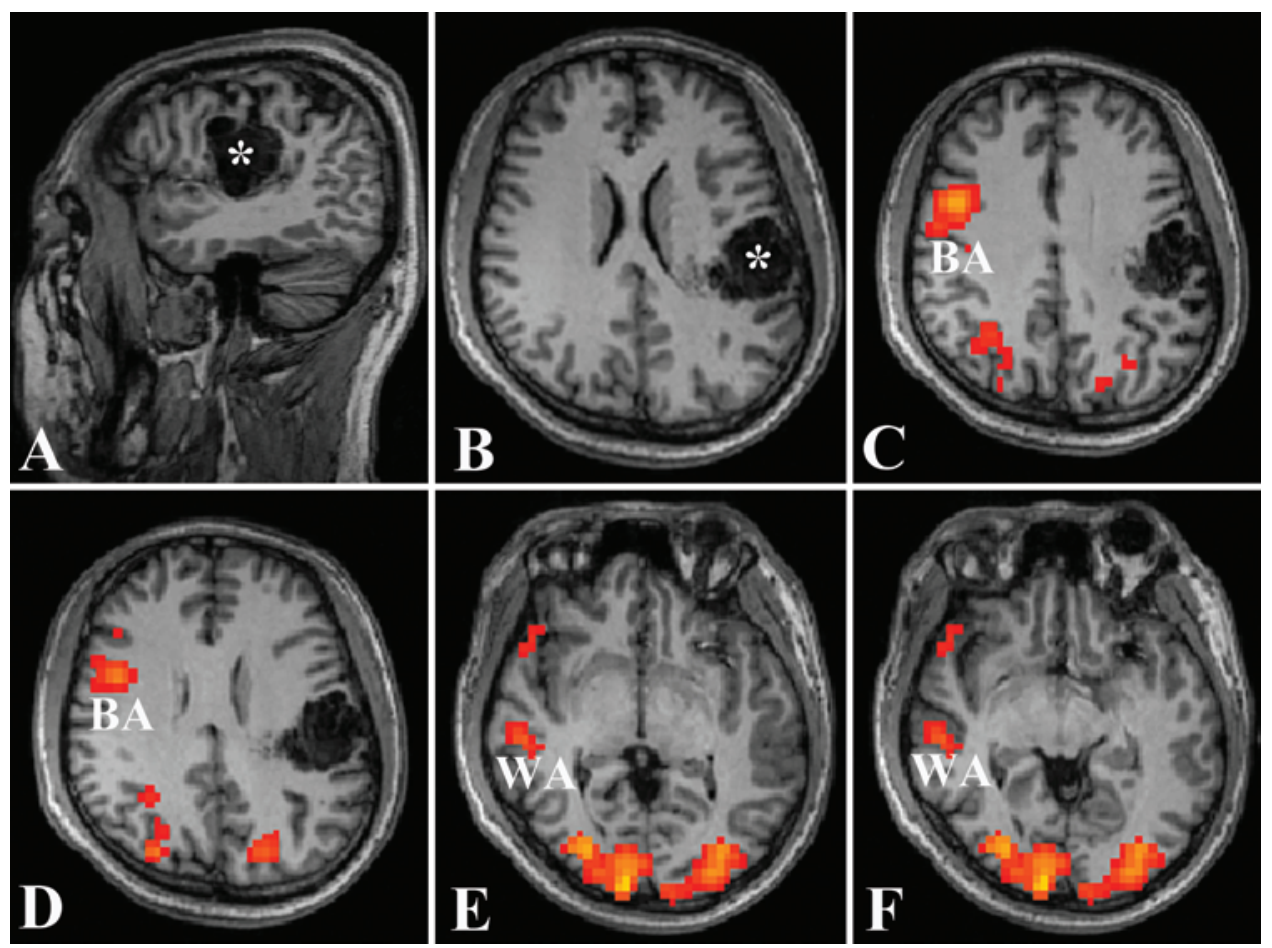

FIG. 1. MR images of a patient in the AVM-BA subgroup. Sagittal (A) and axial (B) T1-weighted MR images reveaing an AVM (asterisk) involving the Broca area. FMR images showing right-sided BOLD signal activation of both the Broca $(\mathbf{C}$ and $\mathbf{D})$ and the Wernicke ( $\mathbf{E}$ and $\mathbf{F}$ ) areas. Figure is available in color online only.

subgroup $(11.8 \%)\left(\chi^{2} 8.683, \mathrm{p}=0.003\right.$, OR $6.094[95 \% \mathrm{CI}$ 1.704-21.794]).

Language disorders were observed in $3(4.8 \%)$ of 63 patients, including 2 patients in the WA subgroup who presented with fluent aphasia and 1 patient in the BA subgroup who presented with nonfluent aphasia. No significant difference in language dysfunction was observed between the 2 subgroups $\left(\chi^{2} 0.020, p=0.888\right)$.

\section{Glioma Group Analysis}

In the glioma group, $6(15.8 \%)$ of the 38 patients showed right-sided BOLD lateralization of the Wernicke area ( $\mathrm{LI}=-0.40 \pm 0.15$; Fig. 3 ), including 2 patients in the BA subgroup and 4 patients in the WA subgroup (Table 1). No patient had right-sided lateralization of the Broca area. Among the 26 patients with a low-grade glioma (WHO Grade I-II), right-sided activation of the Wernicke area was observed in 5 patients (19.2\%), including 2 in the BA subgroup and 3 in the WA subgroup; of the 12 patients with a high-grade glioma (WHO Grade III-IV), only 1 $(8.3 \%)$ in the WA subgroup had right-sided activation of the Wernicke area. No significant difference was detected between low-grade gliomas and high-grade gliomas ( $\mathrm{p}=$ 0.643 , Fisher exact test). Moreover, although the incidence of right-sided lateralization was higher in the WA subgroup (28.6\%) than in the BA subgroup (8.3\%), no significant difference was detected between the 2 subgroups ( $p$ $=0.167$, Fisher exact test). The other factors were also not correlated with right-sided lateralization of BOLD signal activation, including tumor size $(\mathrm{p}=0.430)$ and duration of symptoms $(\mathrm{p}=0.132)$.

Language disorders were observed in 14 (36.8\%) of the
38 patients, including 12 patents in the BA subgroup and 2 patients in the WA subgroup. All of these patients presented with nonfluent aphasia. The incidence of language dysfunction was significantly higher in the BA subgroup $(50.0 \%)$ than in the WA subgroup $(14.3 \%)(\mathrm{p}=0.039$, Fisher exact test). In addition, although the incidence of language dysfunction was higher in patients with a highgrade glioma (5 in 12 [41.7\%]) than in patients with a lowgrade glioma (9 in $26[34.6 \%]$ ), no significant difference was observed ( $\mathrm{p}=0.728$, Fisher exact test).

\section{Differences Between the AVM and Glioma Groups}

Overall, the incidence of right-sided lateralization of BOLD signals was significantly higher in the AVM group $(36.5 \%)$ than in the glioma group $(15.8 \%)\left(\chi^{2} 4.971, \mathrm{p}=\right.$ 0.026, OR 3.097 [95\% CI 1.115-8.433]), and the incidence of aphasia was significantly higher in the glioma group (36.8\%) than in the AVM group (4.8\%) $\left(\chi^{2} 17.426, \mathrm{p}<\right.$ 0.001, OR 11.667 [95\% CI 3.074-44.279]).

\section{Discussion}

Previous studies have demonstrated language reorganization in the setting of various pathological entities, including AVMs, $, 11,13,24$ strokes, ${ }^{26}$ epilepsy,${ }^{10}$ and brain tumors. $7,18,20,25$ Language reorganization in patients with an AVM has been of particular concern, because an AVM located in the language cortex usually does not lead to aphasia unless it has ruptured. However, few studies have compared language cortex reorganization among patients with different pathological entities, especially between patients with an AVM and those with a glioma. Also, most 

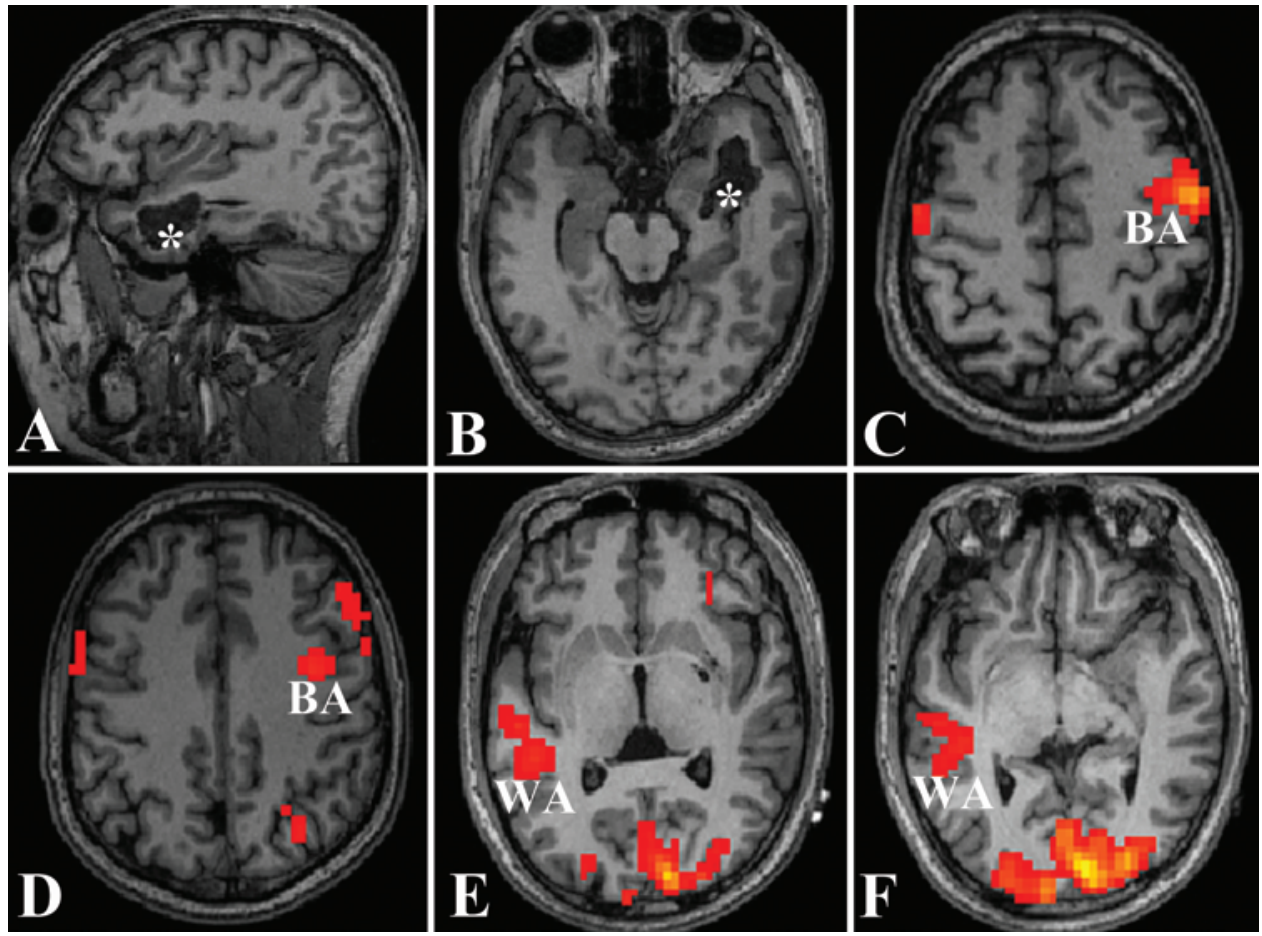

FIG. 2. MR images of a patient in the AVM-WA subgroup. Sagittal (A) and axial (B) T1-weighted MR images revealing an AVM (asterisk) adjacent to the Wernicke area. fMR images showing left-sided activation of the Broca area (C and $\mathbf{D}$ ) and right-sided activation of the Wernicke area ( $\mathbf{E}$ and $\mathbf{F})$. Figure is available in color online only.

previous studies focused on global LIs as a measure of language lateralization, and the Broca and Wernicke areas were seldom discussed separately. Therefore, we conducted this study to compare language cortex reorganization in a large series of patients with an AVM or a glioma, and we analyzed the Broca and Wernicke areas separately.

\section{Modalities for Language Mapping}

Wada testing and electrocortical stimulation mapping (ESM) have long been considered the gold standards for assessing language dominance, but they are both invasive. Meanwhile, fMRI has emerged as the leading noninvasive modality for preoperative language mapping, although its accuracy remains a matter of debate. Some authors have questioned the accuracy of fMRI, especially in patients with an AVM. ${ }^{23}$ They have hypothesized that AVMs can lead to a reduction of cerebral perfusion around extranidal tissues (arterial steal) and result in abnormal fMRI results because BOLD fMRI depends on blood oxygen levels. ${ }^{23}$ However, most studies that focused on comparing fMRI and ESM have shown that fMRI is actually a very sensitive imaging modality, even in patients with vascular malformations ${ }^{3,17}$ For example, Pouratian et al. ${ }^{17}$ analyzed the results of BOLD fMRI and ESM in 7 patients with AVMs and 3 patients with cavernous malformations; they demonstrated a high sensitivity (range 96.2\%-100\%) and an acceptable specificity (range $66.7 \%-69.8 \%$ ) of fMRI. Lee et al. ${ }^{12}$ analyzed fMRI data from 15 patients with AVMs and 7 with cavernous malformations, 8 of whom underwent the Wada tesing. Language lateralization results of fMRI were identical to those of the Wada tests in each of the 8 patients. Also, their results showed that the over- all activation patterns were equivalent in right- and leftdominant patients, and there was a statistically significant increased number of right-hemisphere-activated voxels in the right-sided lateralization cases, which indicates that the right-sided lateralization on fMRI was true rather than an imaging-related artifact. Therefore, considering its high sensitivity and acceptable specificity, fMRI was employed for preoperative language mapping in our department.

\section{Dominant Language Areas on fMRI}

It has been noticed that the Broca and Wernicke areas can be lateralized asymmetrically in the setting of intracranial pathological entities. ${ }^{24}$ In this study, a total of 29 patients had right-sided language lateralization; we observed 24 patients $(82.8 \%)$ whose Broca and Wernicke areas were lateralized asymmetrically, including 18 patients with an AVM and 6 patients with a tumor. Similarly, in the Lee et al..$^{12}$ study, $3(60.0 \%)$ of 5 right-dominant patients had asymmetrically lateralized language areas. The high ratio suggests that studying the global LI alone is inappropriate in the setting of intracranial lesions, which might lead to wrong conclusions regarding language lateralization, because the activated voxels in different hemispheres will offset each other according to the LI formula.

It is interesting to note that a significant difference in BOLD signal activation was observed in patients with an AVM with the nidus in the left frontal area (BA subgroup) and the temporal area (WA subgroup). More specifically, right-sided lateralization of the Broca area was significantly more common in the BA subgroup (26.5\%) than in the WA subgroup (6.9\%) $(\mathrm{p}=0.041)$; likewise, rightsided lateralization of the Wernicke area was significantly 

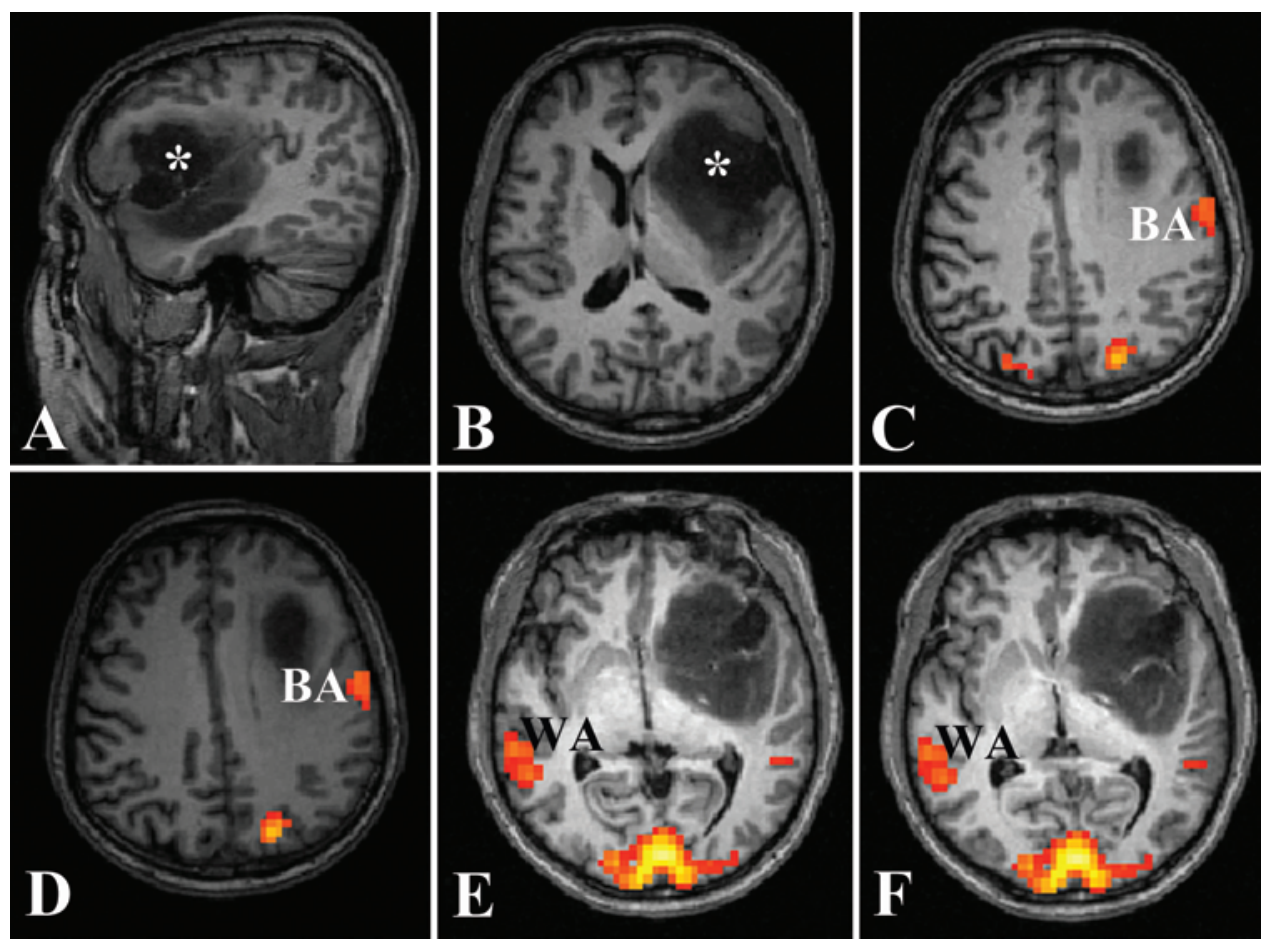

FIG. 3. MR images of a patient in the glioma-BA subgroup. Sagittal (A) and axial (B) T1-weighted MR images revealing an astrocytoma (asterisk) involving the left frontal and temporal lobes. fMR images showing left lateralization of the Broca area (C and $\mathbf{D})$ and right-sided lateralization of the Wernicke area ( $\mathbf{E}$ and $\mathbf{F})$. Figure is available in color online only.

more common in the WA subgroup $(44.8 \%)$ than in the BA subgroup $(11.8 \%)(\mathrm{p}=0.003)$. In other words, a nidus near the Broca area mainly leads to right-sided lateralization of the Broca area, and a nidus near the Wernicke area mainly leads to right-sided lateralization of the Wernicke area. This "mirror phenomenon" suggests that the homologous language areas in the nondominant hemisphere play similar roles when the corresponding language area in the dominant hemisphere is impaired. However, this phenomenon was not observed in the patients with a glioma. We think that the possible reason is that the language areas may have been well reorganized in patients with an AVM but poorly reorganized in the patients with a glioma. In this series, no patient with a glioma had right-sided activation of the Broca area, so this phenomenon was not observed. In a previous study, Wang et al. ${ }^{25}$ analyzed the fMRI findings in 43 patients with a brain tumor (31 in the left hemisphere and 12 in the right hemisphere), and they found that the tumors in the left frontal region affected both the Broca and the Wernicke areas, whereas tumors in the left temporal lobe affected mainly the Wernicke area. The difference between the 2 studies might be attributable to statistical methods and different pathologies, because their brain tumor diagnoses were based on MR images rather than pathology examinations.

A difference in patterns of language reorganization may be expected between high-grade and low-grade gliomas. In general, growth and progression of a low-grade glioma take longer than a high-grade glioma. Low-grade gliomas in the left hemisphere can cause slow destruction of language areas while leaving language function relatively intact as other brain regions develop or "rewire" to compensate for the loss of language functions in primary language areas. However, high-grade gliomas grow fast, and it is more common for them to cause function deficits. ${ }^{6,14}$ In this study, the incidence of language dysfunction was higher in patients with a high-grade glioma (41.7\%) than in patients with a low-grade glioma (34.6\%), and right-sided lateralization was more common with low-grade gliomas $(19.2 \%)$ than with high-grade gliomas $(8.3 \%)$. However, no significant difference was detected in either the clinical presentations or the fMRI findings. We believe that the possible reason is the sample size. Although we enrolled a large series of patients, the right-sided lateralization of language areas in the glioma group was rare. Therefore, the number of patients with right-sided activation shown with fMRI was relatively too small to study the difference between high-grade and low-grade gliomas, which may lead to false-negative findings.

\section{Comparison Between the AVM and Glioma Groups}

In this study, right-sided lateralization of BOLD signal activations was observed in both the AVM group and the glioma group, suggesting that language cortex reorganization may occur in the setting of AVMs and gliomas. The incidence of right-hemisphere lateralization was significantly higher in the AVM group than in the glioma group $(\mathrm{p}=0.026)$. Meanwhile, language dysfunction was more common in the glioma group (36.8\%) than in the AVM group $(4.8 \%)(\mathrm{p}<0.001)$. All these results show that patients with an AVM have a greater capacity for language cortex reorganization than patients with a glioma. 
We speculate that this difference in patterns of language cortex reorganization is a result of several reasons. First and foremost, the stage of lesion development might be a factor that affects the potential for language reorganization. Although the exact period in which cerebral AVMs occur is still controversial, and some studies have suggested that they develop postnatally, there is a consensus that AVMs develop in early life..$^{9,13}$ On the contrary, gliomas are acquired and mainly develop in adult patients. Also, the patients with a glioma who were enrolled in this study were all adults. Thus, we believe that the probable reason is that the brain possesses greater plasticity in its immature than in its mature state, which has been demonstrated by animal experiments that showed that the recovery of function is generally greater when brain damage occurs early in life rather than in adulthood. ${ }^{16}$ Consequently, when AVMs develop in the anatomical site of eloquent cortex in early life, neuroplasticity will result in cortical reorganization of the functional areas, with displacement to homologous regions on the contralateral side. Therefore, language function will seldom be impaired. ${ }^{1}$ Second, the duration of illness might be another reason. Unruptured AVMs are a chronic disease with a long duration of illness, whereas the course of tumors is relatively short. Thus, we speculate that the brain has a greater ability to reorganize in the setting of a chronic disease rather than of an acute disease. In addition, the differential effect on language lateralization may be a result of histopathological differences between AVMs and tumors. AVMs are characterized by massive abnormal vessels, and there is usually normal brain parenchyma within the nidus. Also, a mass effect seldom occurs unless an AVM has ruptured. On the contrary, although gliomas may have abnormal tumor vessels, they are different from those of AVMs, which are composed of feeding arteries, malformed vessels, and draining veins. Also, there is no normal brain parenchyma within the tumors, and a mass effect usually exists. We believe that all of these factors may result in the difference in patterns of language cortex reorganization in patients with a cerebral AVM and those with a glioma.

\section{Limitations of Study}

The present study has several limitations. First, it was a retrospective research study with limitations inherent to such a study design. Second, although we enrolled a large series of 63 patients with an AVM and 38 patients with a glioma, the sample size of the glioma group is too small to study the difference between high-grade and low-grade gliomas, which may lead to false-negative findings. Third, this was an fMRI study, and confirmatory tests (ESM and Wada testing) were not conducted, but they would have been useful for corroborating the results. Furthermore, only a silent reading task was performed by the patients in this study. Although activations of both the Broca and the Wernicke areas were observed in all the patients, multiple tasks can activate different aspects of the language network, and the results of multiple tasks may make our conclusions more accurate and more reliable. Overall, additional studies with larger series of patients undergoing both multiple-task fMRI and confirmatory tests (ESM and Wada testing) are needed to confirm our conclusions.

\section{Conclusions}

Patients with a cerebral AVM and those with a glioma had right-sided lateralization of BOLD signal activations, which suggests that language cortex reorganization may occur with both diseases. However, the potential for reorganization in patients with a glioma seems to be insufficient compared with that in patients with an AVM, as suggested by both clinical manifestations and fMRI findings. Moreover, our study results seem to indicate that in patients with an AVM, a nidus near the Broca area mainly leads to right-sided activation of the Broca area, whereas a nidus near the Wernicke area mainly leads to right-sided activation of the Wernicke area. Patients with a glioma did not show this phenomenon, and no significant difference in right-sided lateralization was detected between lowgrade and high-grade gliomas.

\section{References}

1. Alkadhi H, Kollias SS, Crelier GR, Golay X, Hepp-Reymond $\mathrm{MC}$, Valavanis A: Plasticity of the human motor cortex in patients with arteriovenous malformations: a functional MR imaging study. AJNR Am J Neuroradiol 21:1423-1433, 2000

2. Arteriovenous Malformation Study Group: Arteriovenous malformations of the brain in adults. N Engl J Med 340:1812-1818, 1999

3. Cannestra AF, Pouratian N, Forage J, Bookheimer SY, Martin NA, Toga AW: Functional magnetic resonance imaging and optical imaging for dominant-hemisphere perisylvian arteriovenous malformations. Neurosurgery 55:804-814, 2004

4. Chang EF, Wang DD, Perry DW, Barbaro NM, Berger MS: Homotopic organization of essential language sites in right and bilateral cerebral hemispheric dominance. J Neurosurg 114:893-902, 2011

5. Choi JH, Mohr JP: Brain arteriovenous malformations in adults. Lancet Neurol 4:299-308, 2005

6. Dronkers NF, Wilkins DP, Van Valin RD Jr, Redfern BB, Jaeger JJ: Lesion analysis of the brain areas involved in language comprehension. Cognition 92:145-177, 2004

7. Duffau $\mathrm{H}$ : The huge plastic potential of adult brain and the role of connectomics: new insights provided by serial mappings in glioma surgery. Cortex 58:325-327, 2014

8. Fuller GN, Scheithauer BW: The 2007 Revised World Health Organization (WHO) Classification of Tumours of the Central Nervous System: newly codified entities. Brain Pathol 17:304-307, 2007

9. Gross BA, Du R: Natural history of cerebral arteriovenous malformations: a meta-analysis. J Neurosurg 118:437-443, 2013

10. Janszky J, Mertens M, Janszky I, Ebner A, Woermann FG: Left-sided interictal epileptic activity induces shift of language lateralization in temporal lobe epilepsy: an fMRI study. Epilepsia 47:921-927, 2006

11. La Piana R, Klein D, Cortes M, Tampieri D: Speech reorganization after an AVM bleed cured by embolization. A case report and review of the literature. Interv Neuroradiol 15:456-461, 2009

12. Lee DJ, Pouratian N, Bookheimer SY, Martin NA: Factors predicting language lateralization in patients with perisylvian vascular malformations. Clinical article. J Neurosurg 113:723-730, 2010

13. Lehéricy S, Biondi A, Sourour N, Vlaicu M, du Montcel ST, Cohen L, et al: Arteriovenous brain malformations: is functional MR imaging reliable for studying language reorganization in patients? Initial observations. Radiology 223:672-682, 2002 
14. Mbwana J, Berl MM, Ritzl EK, Rosenberger L, Mayo J, Weinstein S, et al: Limitations to plasticity of language network reorganization in localization related epilepsy. Brain 132:347-356, 2009

15. Mohr JP: Brain arteriovenous malformations: children and adults. Stroke 36:2060-2061, 2005

16. Passingham RE, Perry VH, Wilkinson F: The long-term effects of removal of sensorimotor cortex in infant and adult rhesus monkeys. Brain 106:675-705, 1983

17. Pouratian N, Bookheimer SY, Rex DE, Martin NA, Toga AW: Utility of preoperative functional magnetic resonance imaging for identifying language cortices in patients with vascular malformations. J Neurosurg 97:21-32, 2002

18. Rösler J, Niraula B, Strack V, Zdunczyk A, Schilt S, Savolainen P, et al: Language mapping in healthy volunteers and brain tumor patients with a novel navigated TMS system: evidence of tumor-induced plasticity. Clin Neurophysiol 125:526-536, 2014

19. Rutten GJ, Ramsey NF, van Rijen PC, Alpherts WC, van Veelen CW: FMRI-determined language lateralization in patients with unilateral or mixed language dominance according to the Wada test. Neuroimage 17:447-460, 2002

20. Sanai N, Mirzadeh Z, Berger MS: Functional outcome after language mapping for glioma resection. N Engl J Med 358:18-27, 2008

21. Tan LH, Feng CM, Fox PT, Gao JH: An fMRI study with written Chinese. Neuroreport 12:83-88, 2001

22. Tan LH, Liu HL, Perfetti CA, Spinks JA, Fox PT, Gao JH: The neural system underlying Chinese logograph reading. Neuroimage 13:836-846, 2001

23. Ulmer JL, Hacein-Bey L, Mathews VP, Mueller WM, DeYoe EA, Prost RW, et al: Lesion-induced pseudo-dominance at functional magnetic resonance imaging: implications for preoperative assessments. Neurosurgery 55:569-581, 2004

24. Vikingstad EM, Cao Y, Thomas AJ, Johnson AF, Malik GM,
Welch KM: Language hemispheric dominance in patients with congenital lesions of eloquent brain. Neurosurgery 47:562-570, 2000

25. Wang L, Chen D, Yang X, Olson JJ, Gopinath K, Fan T, et al: Group independent component analysis and functional MRI examination of changes in language areas associated with brain tumors at different locations. PLoS ONE 8:e59657, 2013

26. Winhuisen L, Thiel A, Schumacher B, Kessler J, Rudolf J, Haupt WF, et al: Role of the contralateral inferior frontal gyrus in recovery of language function in poststroke aphasia: a combined repetitive transcranial magnetic stimulation and positron emission tomography study. Stroke 36:1759-1763, 2005

\section{Author Contributions}

Conception and design: Zhao, Deng. Acquisition of data: Y Zhang, S Wang, Wu, D Zhang, R Wang, J Wang. Analysis and interpretation of data: Deng, Y Zhang, S Wang, Wu, D Zhang, $\mathrm{R}$ Wang, J Wang. Drafting the article: Deng. Critically revising the article: Zhao, Y Zhang, L Xu, B Wang, S Wang, D Zhang, R Wang. Reviewed submitted version of manuscript: Zhao, Y Zhang, L Xu, B Wang, S Wang, Wu, D Zhang, R Wang, J Wang. Approved the final version of the manuscript on behalf of all authors: Zhao. Statistical analysis: L Xu, B Wang. Administrative/technical/material support: L Xu, B Wang. Study supervision: Zhao, S Wang.

\section{Correspondence}

Jizong Zhao, Department of Neurosurgery, Beijing Tiantan Hospital, Capital Medical University, No. 6 Tiantan Xili, Chongwen District, Beijing 100050, China. email: zhaojz@public.bta. net.cn. 